\title{
Probabilistic forecasting of ionospheric scintillation and GNSS receiver signal tracking performance at high latitudes
}

\author{
Paul Prikryl ${ }^{1,{ }^{\star}}$, Veettil Sreeja ${ }^{2}$, Marcio Aquino ${ }^{2}$, Periyadan T. Jayachandran ${ }^{3}$ \\ ${ }^{1}$ Communications Research Centre, Ottawa, ON, Canada \\ ${ }^{2}$ University of Nottingham, Nottingham Geospatial Institute, Nottingham, United Kingdom \\ ${ }^{3}$ University of New Brunswick, Physics Department, Fredericton, NB, Canada
}

\author{
Article history \\ Received December 20, 2011; accepted January 13, 2012. \\ Subject classification: \\ Scintillation, Space weather forecasting, GNSS tracking performance.
}

\begin{abstract}
At high latitudes, phase scintillation occurs predominantly on the dayside in the ionospheric footprint of the magnetospheric cusp, and in the nightside auroral oval. A new technique of probabilistic forecasting of phase scintillation occurrence relative to the arrival time of high-speed solar wind from coronal holes and interplanetary coronal mass ejections has recently been proposed [Prikryl et al. 2012]. Cumulative probability distribution functions for the phase-scintillation occurrence that are obtained can be specified for low (below-median) and high (above-median) values of various solar wind plasma parameters. Recent advances in modeling of high-speed solar wind and coronal mass ejections, combined with the probabilistic forecasting of scintillation, will lead to improved operational space weather forecasting applications. Scintillation forecasting and mitigation techniques need to be developed to avoid potential costly failures of technology-based Global Navigation Satellite Systems in the near future, in particular during the upcoming solar maximum. The Global Navigation Satellite Systems receiver-tracking performance during severe scintillation conditions can be assessed by the analysis of receiver phase-locked-loop jitter. Tracking jitter maps [Sreeja et al. 2011] offers a potentially useful tool to provide users with expected tracking conditions, if based on scintillation predictions as proposed above. Scintillation indices are obtained from L1 GPS data collected with the Canadian High Arctic Ionospheric Network. Combined with high rate amplitude and phase data, they can be used as input to receiver tracking models to develop scintillation mitigation techniques.
\end{abstract}

\section{Introduction}

Global Navigation Satellite Systems (GNSS), such as the US Global Positioning System (GPS), the Russian GNSS (GLONASS) and the EU Galileo, have become indispensable for precise positioning and time keeping for the support of a wide range of civilian and military applications. The main threat to reliable and safe operation of modern technology that relies on GNSS is the variable propagation conditions that are encountered by radio signals as they pass through the ionosphere. Variable ionospheric delays and signal scintillation can affect the performance of radio communications and navigation systems, and can severely degrade GPS positional accuracy. Scintillation, rapid signal amplitude fading, and phase fluctuation, are phenomena of great concern at low and high latitudes. As pointed out by Conker et al. [2003], to determine the effects of scintillation on GNSS receivers, including the GPS / SatelliteBased Augmentation System (SBAS), "both predictions of scintillation activity in the ionosphere and residual effects of this activity after processing by a receiver" must be considered. These authors estimated the scintillation effects on the availability of GPS and SBAS for L1 $\left(1575.42^{\circ} \mathrm{MHz}\right) \mathrm{C} / \mathrm{A}$ and L2 $\left(1227.60^{\circ} \mathrm{MHz}\right)$ semicodeless receivers. In the present study, the benefits of a probabilistic forecasting of phase scintillation to applications relying on robust and accurate phase signal tracking are addressed.

Ionospheric scintillation, which is seen as fluctuations of radio signal amplitude and phase that are caused by ionospheric irregularities [Aarons 1982, Basu et al. 1998], is most intense at low latitudes during post sunset hours [Basu et al. 2002], and at high latitudes in the nightside auroral oval and on the dayside in the region called the cusp [Basu et al. 1998, Spogli et al. 2009, Prikryl et al. 2011a]. At high latitudes, ionospheric scintillation is largely controlled by the process of coupling between the solar wind, including the interplanetary magnetic field, and the Earth magnetic field. In the nightside auroral oval, intense electric currents (auroral electrojets), various plasma instabilities, and energetic particle precipitation result in steep plasma density gradients and irregularities. It has been shown that scintillation strongly affects GPS receiver performance in the 


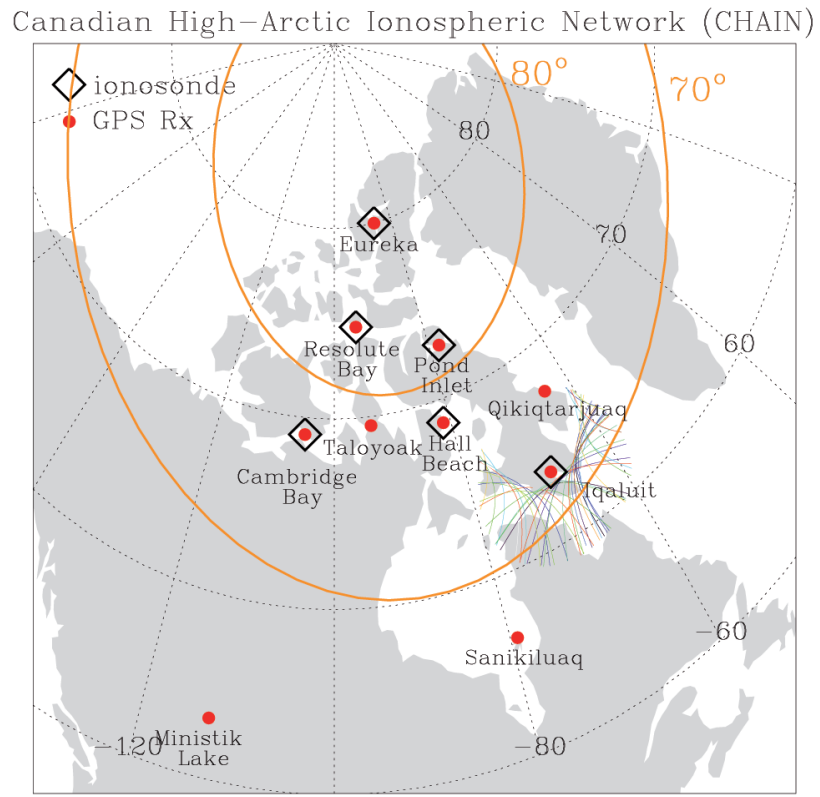

Figure 1. Canadian High Arctic Ionospheric Network (CHAIN). The loci of IPPs at $350 \mathrm{~km}$ altitude for all GPS satellites, as seen from Iqaluit at elevation angles above $30^{\circ}$ during one day. The CGM latitudes $70^{\circ}$ and $80^{\circ}$ (yellow) are superposed over the geographic grid in the dotted line.

auroral zone [e.g., Skone and de Jong 2000].

In the cusp, just poleward of the dayside auroral oval, where intense ionospheric convection and formation of patches of enhanced ionization occur, scintillation occurrence is particularly enhanced. Scintillation occurrence is more seasonally dependent in the cusp than in the nightside auroral oval [Prikryl et al. 2011a], which is characterized by annual and semi-annual variations, respectively. It is also strongly enhanced during intense solar wind events, as either solar wind co-rotating interaction regions (CIRs), or interplanetary coronal mass ejections (ICMEs). ICMEs and CIRs on the leading edge of high-speed streams (HSSs) from coronal holes are major causes of ionospheric disturbances. The strongest of these solar wind events result in major geomagnetic storms [e.g., Borovsky and Denton 2006, Richardson et al. 2006] that have a global impact on the ionosphere. Based on a statistical study of scintillation occurrence during such events from the years 2008 to 2010, a new method of probabilistic forecasting of phase scintillation was proposed [Prikryl et al. 2012]. A summary of this method using a larger number of events is presented in the present study.

Scintillation indices, $S_{4}$ and $\sigma_{\Phi}$, are widely used as the measures of amplitude and phase scintillation, respectively. The $S_{4}$ index is the standard deviation of the received power normalized by its mean value, and the phase scintillation index $\sigma_{\Phi}$ is the standard deviation of the detrended phase using a filter in the receiver with a $0.1 \mathrm{~Hz}$ cut-off frequency. However, as pointed out by Aquino et al. [2007], these scintillation indices do not fully characterize the actual instantaneous values of phase and amplitude fluctuations that affect GNSS receiver performance. They combined scintillation indices and high rate GNSS phase and amplitude data with state-of-the-art receiver tracking models, to study receiver tracking performance under scintillation conditions. This strategy was also applied for an intense scintillation event at low latitudes [Sreeja et al. 2011], where the variance of the phase-locked-loop (PLL) tracking error (tracking jitter) of a GPS receiver was used to construct maps that can provide an indication of tracking conditions for that particular receiver. In this study, tracking-jitter maps are obtained for a highlatitude scintillation event.

\section{The Canadian High Arctic Ionospheric Network}

At present, the Canadian High Arctic Ionospheric Network (CHAIN) [Jayachandran et al. 2009] consists of ten specialized GPS receivers and six Canadian Advanced Digital Ionosondes that are distributed in the auroral oval and the polar cap (Figure 1). The latitudes and longitudes of the stations can be found elsewhere [e.g., Prikryl et al. 2011a, their Table 1]. The receivers are GPS ionospheric scintillation and total electron content (TEC) monitors (GISTMs) GSV 4004B [Van Dierendonck and Arbesser-Rastburg 2004]. The GISTMs consist of a NovAtel OEM4 dual frequency receiver with special firmware specifically configured to measure and log the power and phase of the GPS L1 signal at a high sampling rate $(50 \mathrm{~Hz})$. The receiver computes ionospheric TEC from the GPS L1 and L2 signals. The GSV 4004B can also automatically compute and record $S_{4}$ and $\sigma_{\Phi}$ indices; 1-min values are used in the present study. Each receiver can track and report scintillation and TEC measurements simultaneously from up to 10 GPS satellites in view.

\section{Occurrence of GPS phase scintillation at high lat- itudes}

A scintillation climatology study for the solar minimum years of 2008 and 2009 showed that phase scintillation, as a function of magnetic local time and geomagnetic latitude, primarily occurs in the night-side auroral oval and on the dayside in the ionospheric footprint of the cusp [Prikryl et al. 2011a]. As expected, these scintillation regions shift in latitude in response to varying geomagnetic activity. We have extended the climatology data set to 4 years. Figure 2a, b shows the maps of the percentage occurrence of phase scintillation with $\sigma_{\Phi}$ that exceeds 0.1 radians as a function of magnetic local time (MLT) and corrected geomagnetic (CGM) latitude for 1,272 quiet and 189 disturbed days selected using the threshold of 3-h occurrence of $K p>2$ 

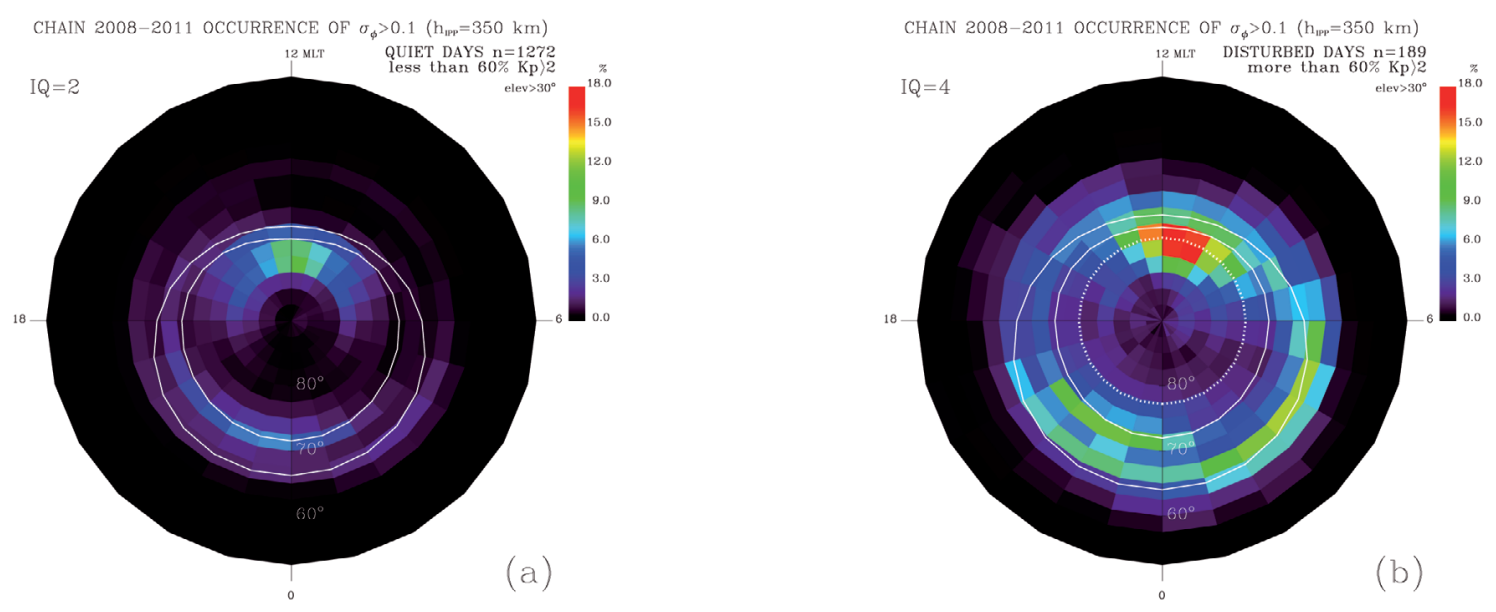

Figure 2. Phase scintillation occurrence maps for geomagnetically quiet (a) and moderately disturbed (b) days.
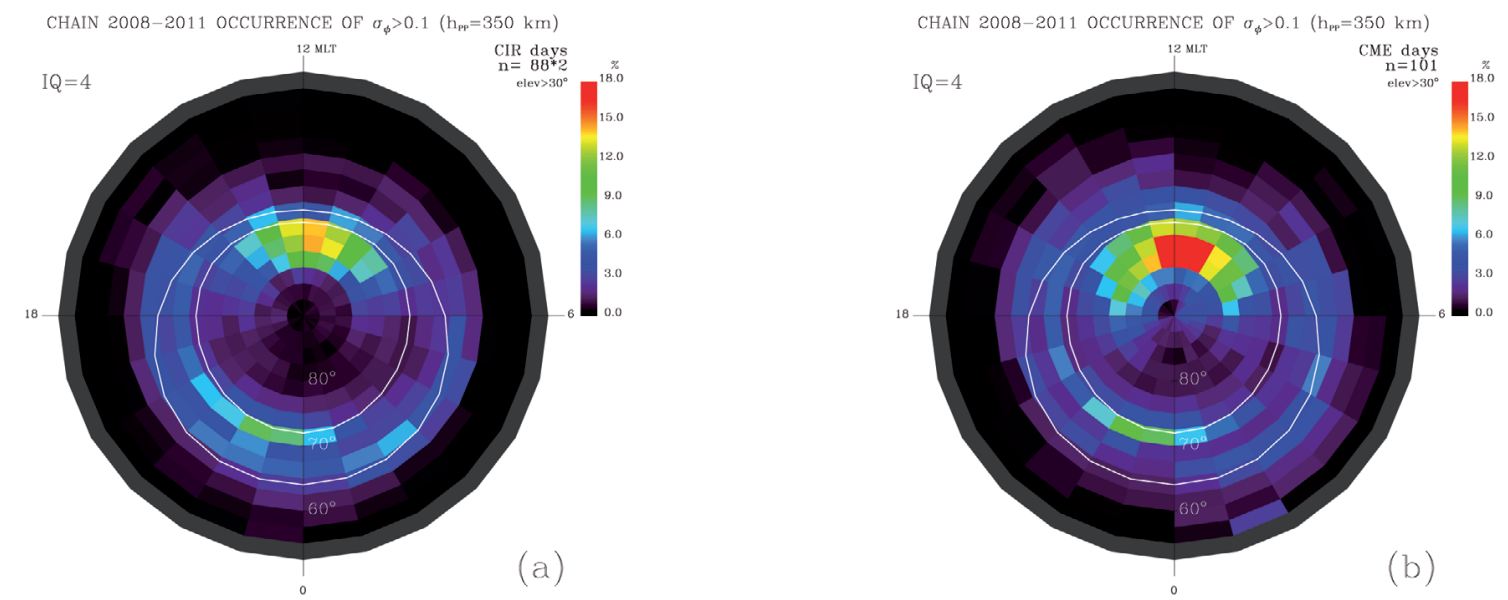

Figure 3. Phase scintillation occurrence maps for days of CIRs/HSSs (a) and ICMEs (b).

for more than $60 \%$ of the day. The boundaries of the Feldstein statistical auroral oval for quiet and moderately disturbed conditions are superposed on the maps [Holtzworth and Meng 1975], indicated by the value of the IQ parameter. A dotted concentric circle in Figure $2 \mathrm{~b}$ shows the latitude of Cambridge Bay. The Cambridge Bay dataset samples the poleward edge of the auroral oval in the night [Prikryl et al. 2010], but it is representative of the cusp during the day. The Cambridge Bay data time series are used to obtain cumulative probability distribution functions for the phase scintillation occurrence for the days before and after the arrivals of CIR/HSSs and ICMEs.

Figure 3a, b shows scintillation occurrence maps for the first two days of HSS / CIRs and for ICME days, respectively. In total, $88 \mathrm{HSS} / \mathrm{CIRs}$ with maximum solar wind velocity exceeding $500 \mathrm{~km} / \mathrm{s}$ are included. The updated catalog of near-Earth ICMEs [Richardson and Cane 2010] includes 57 ICME events from 2008 to 2011 (101 days in total). However, most of these ICMEs did not cause significant geomagnetic storms [e.g., Prikryl et al. 2011b]. Scintillation occurred predominantly in the cusp for both datasets, but the occurrence was higher for ICME days than for HSS / CIR days. This is opposite to the 2008 to 2010 results [Prikryl et al. 2012] and it is a sign that many more significant ICMEs occurred in 2011. Also, comparing the total numbers of HSS / CIRs and ICMEs, they increased by $114 \%$ and $33 \%$, respectively, from 2010 to 2011 .

\section{The superposed-epoch analysis of geomagnetic and phase scintillation indices}

Arrivals of HSSs originating from coronal holes are followed by significantly enhanced phase scintillation occurrence, particularly in the cusp, which usually continues for a few days. To obtain the mean response of the scintillation relative to a new CIR arrival time, a superposed-epoch analysis on time series of scintillation occurrence (Figure 4a) and solar wind parameters was applied, using the key times of 88 CIRs. Figure 4a shows that the mean phase scintillation occurrence steeply rises at the CIR arrival day and tapers off a few days later, which is similar to the mean $K p$ index and the quasi-invariant index, QI [Osherovich et al. 1999]. Also, the average number of GPS cycle slips, which is nearly zero a few days before the CIR key day, increases at the 

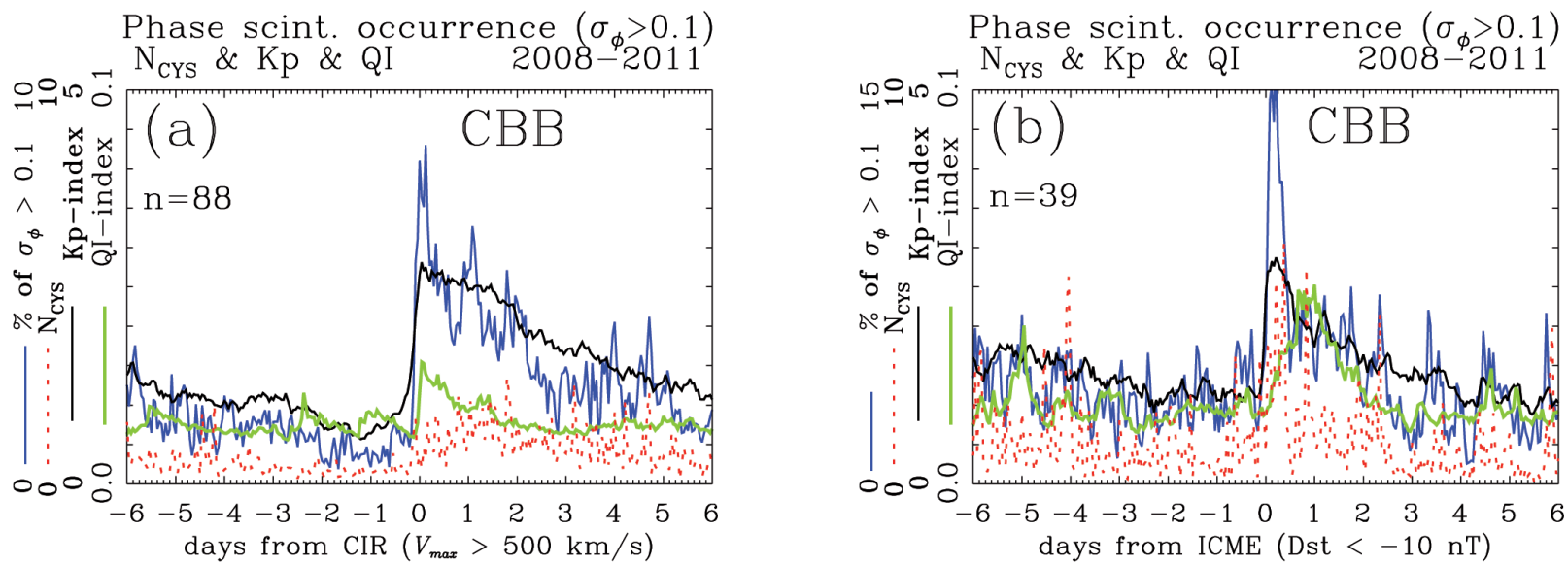

Figure 4. Superposed epoch analysis of QI, Kp, number of cycle slips $N_{c y s}$ and phase scintillation occurrence. The time series are keyed by the start times of CIRs (a) and ICMEs (b).

CIR interface and remains elevated during the HSS. The one-day periodicity in scintillation is due to the Cambridge Bay cusp location and is only partly smoothed out in this relatively small statistical sample of 88 CIRs.

Figure $4 \mathrm{~b}$ shows the results of the superposedepoch analysis for 39 ICMEs for which the Dst index dropped below $-10 \mathrm{nT}$, eliminating the weakest ICMEs. The QI increases more gradually but to higher values than for CIRs. This is because many ICMEs are actually magnetic clouds, and the ratio of the magnetic pressure to ram pressure that the QI represents is higher inside magnetic clouds. The phase scintillation occurrence peaks at $\sim 15 \%$ and the mean number of cycle slips peaks at more than $5 / \mathrm{h}$ on the day of the ICME arrival. A cycle slip [Horvath and Crozier 2007] is defined here as a jump in differential phase TEC of more than or equal to 1.5 TEC Units in $1 \mathrm{~s}$ (1 TEC Unit corresponds to $10^{16}$ electrons $/ \mathrm{m}^{2}$ ). If the superposedepoch analysis is further limited to only 15 of the strongest ICMEs (Dst $<-30 \mathrm{nT})$, the peak mean scintillation occurrence, the mean number of cycle slips, and the $K p$ index are $22 \%, 15$ and 4.3, respectively.

5. Probabilistic forecasting of phase scintillation in the cusp based on the superposed-epoch analysis of solar wind CIRs and ICMEs

McPherron and Siscoe [2004] and McPherron and Weygand [2006] demonstrated a new promising technique to forecast geomagnetic disturbance indices relative to the stream interface between the fast and slow solar wind. In particular, they showed geoeffectiveness of what they called co-rotating compression ridges, their reference to CIRs. This method is readily applicable to other manifestations of space weather in the geospace environment that are sensitive to arrivals of HSSs, including ionospheric scintillation.

Figure 5a shows the cumulative probability distribution function (PDF) for the phase scintillation oc- currence at Cambridge Bay for the days before and after the major CIR/HSSs arrivals. The probability that phase scintillation will exceed a given value is shown, as plotted on the abscissa. The PDFs are stepped by 2-day intervals from 6 days before and 6 days after CIRs. Figure $5 \mathrm{~b}$ shows the PDFs for days before and after ICMEs. The probabilities are clearly highest for the first two days after CIR and ICME arrivals.

These PDFs can be used to develop high-latitude scintillation forecasting techniques [Prikryl et al. 2012]; e.g., scintillation occurrence in the cusp relative to the stream interface between the fast and slow solar wind and/or shocks associated with CIRs and ICMEs. To gain further practical application of the probabilistic forecasting of scintillation occurrence, tracking-jitter maps can be constructed to assess GNSS receiver signal-tracking performance under anticipated scintillation conditions [Sreeja et al. 2011].

\section{Tracking-jitter maps}

In a GNSS receiver, the PLL aims to minimize the error between the input phase and its estimated phase output. It is the magnitude of this error that determines the ability of the loop to remain locked. The variance of the error at the output of the PLL (the tracking-jitter variance) increases during scintillation, and hence is a good measure of the effects of scintillation on the receiver. The tracking-jitter variance at the output of the PLL can be calculated using the scintillation sensitive tracking model of Conker et al. [2003]. The formula for the GPS L1 carrier PLL accounts for the effects of scintillation on the input phase and computes the trackingjitter variance $\left(\sigma_{\phi}^{2}\right)$, in radians ${ }^{2}$, as [Conker et al. 2003]:

$$
\sigma_{\phi}^{2}=\sigma_{\phi_{S}}^{2}+\sigma_{\phi_{T}}^{2}+\sigma_{\phi_{o s c}}^{2}
$$

where $\sigma_{S}^{2}, \sigma_{T}^{2}$ and $\sigma_{O S C}^{2}$ are, respectively, the error variance components relating to the phase scintillation, the 

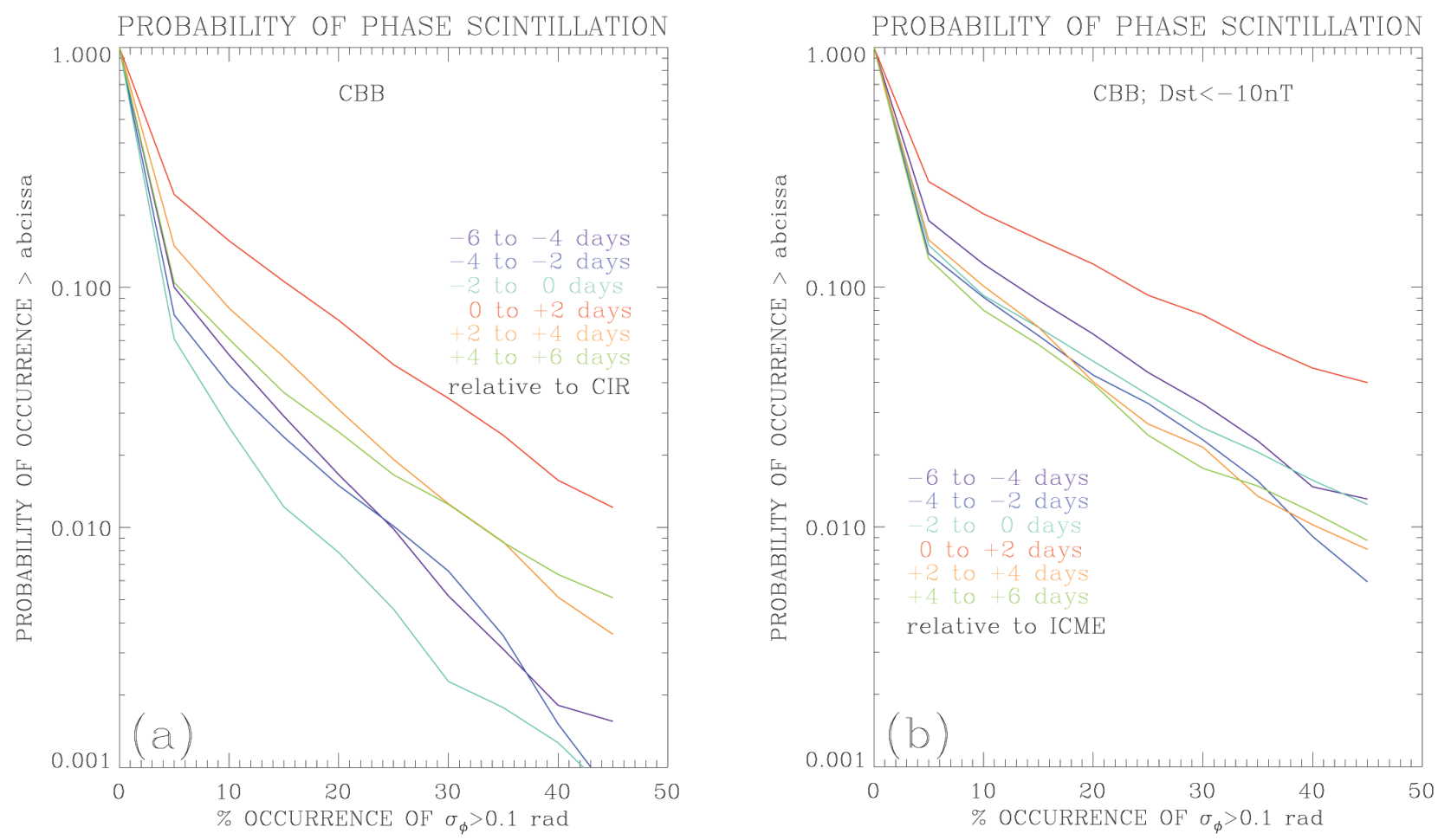

Figure 5. Cumulative probability distribution functions for the phase scintillation occurrence for Cambridge Bay, stepped by 2-day intervals before and after CIRs $(\mathrm{n}=88)$ (a) and ICMEs (Dst $<-10 \mathrm{nT} ; \mathrm{n}=39)$ (b).

thermal noise (amplitude scintillation), and the oscillator noise (assumed to be 0.01 radians $^{2}$ in the receiver).

In Equation (1), the amplitude scintillation is modeled as an increase in the thermal noise, related to the decrease in the received signal power, in radians ${ }^{2}$, as [Conker et al. 2003]:

$$
\sigma_{\phi_{T}}^{2}=\frac{B_{n}\left[1+\frac{1}{2 \eta\left(c / n_{0}\right)_{L 1-C / A}\left(1-2 S_{4}^{2}(L 1)\right)}\right]}{\left(c / n_{0}\right)_{L 1-C / A}\left(1-2 S_{4}^{2}(L 1)\right)}
$$

where $B_{n}$ is the L1 third-order PLL one-sided bandwidth (equal to $10 \mathrm{~Hz}$ in this study), $\left(c / n_{0}\right)_{L 1-C / A}$ is the fractional form of the signal-to-noise density ratio, equal to $10^{0.1 \mathrm{C} / \mathrm{No}}, \eta$ is the predetection integration time (equal to $0.02 \mathrm{~s}$ in this study), and $S_{4}(\mathrm{~L} 1)$ is the amplitude scintillation index at $\mathrm{L} 1$ frequency.

The phase scintillation component is modeled, in radians $^{2}$, as [Conker et al. 2003]:

$$
\sigma_{\phi_{S}}^{2}=\frac{\pi T}{k f_{n}^{p-1} \sin \left[\frac{[2 k+1-p] \pi}{2 k}\right]}
$$

where $T$ is the spectral strength of the phase power spectrum density (PDS) at $1 \mathrm{~Hz}, p$ is the spectral slope of the phase PSD, $k$ is the order of the PLL (equal to 3 in this study), and $f_{n}$ is the loop natural frequency (equal to $1.91 \mathrm{~Hz}$ ).

For this study, the one minute scintillation indices and the high data rate $(50-\mathrm{Hz}$ phase and amplitude fluc- tuations) for the GPS L1 C/ A signal recorded by the receivers in the CHAIN network on November 1, 2011, were considered. A satellite elevation angle cut-off of $30^{\circ}$ was applied to the data to reduce the impact of non-scintillation-related effects on the tracking errors (e.g., induced by multipath). The latitude of the ionospheric pierce point (IPP) for the different satellite-to-receiver links was calculated every $1 \mathrm{~min}$, assuming a single-shell ionospheric model at an altitude of $350 \mathrm{~km}$. The PLL jitter for the different satellites in view with elevation angles greater than $30^{\circ}$ at each epoch were calculated using the equations above. These PLL jitter relate to the PLL tracking error assumed in the slant direction of the line of sight between receiver and satellite. For practical use of the proposed PLL jitter maps it is necessary that a verticalised version of this error is represented, so that the user can estimate the error for any specific line of sight by applying an appropriate mapping function. This mapping function, as explained in Sreeja et al. [2011], is the subject of ongoing research funded by the UK Engineering and Physical Sciences Research Council (EPSRC). For the construction of the map presented in the present study, however, an approximation was used to convert the slant PLL jitter to vertical PLL jitter, by assuming a standard mapping function $\cos \chi$, where $\chi$ is the zenith angle at the IPP. The values of the verticalised PLL jitter were then gridded in bins with a resolution of $1 \mathrm{~min}$ in time and $0.5^{\circ}$ in IPP latitude, to produce the PLL jitter map of Figure 7 in the next section. 


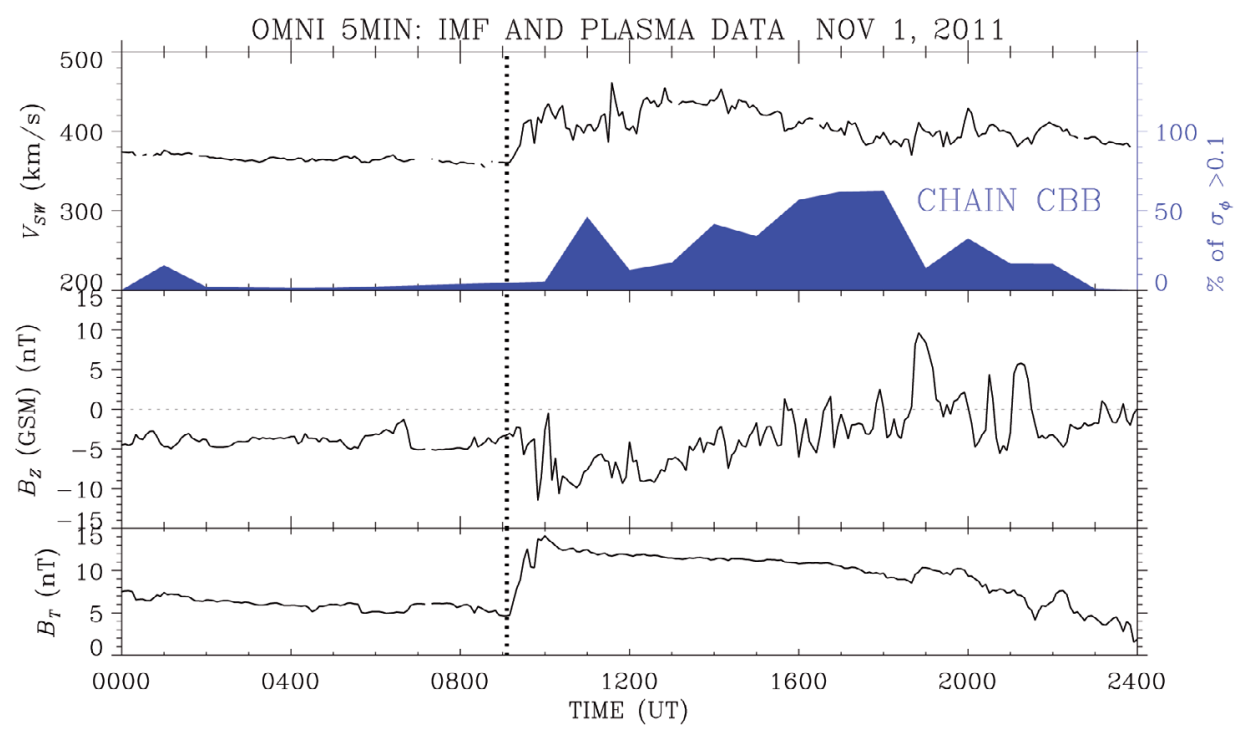

Figure 6. Solar wind magnetic field and plasma parameters from OMNIWeb dataset combining available solar wind monitor data projected to the nose of the Earth bow shock. The hourly percent occurrence of $\sigma_{\Phi}>0.1 \mathrm{rad}$ from Cambridge Bay is highlighted in blue.

\section{The event of November 1,2011}

Figure 6 shows 5 -min averages of the solar wind velocity, $V_{s w}$, the interplanetary magnetic field (IMF) $B_{Z}$ component in geocentric-solar-magnetospheric (GSM) coordinates, and the magnetic field magnitude, $B_{T}$,

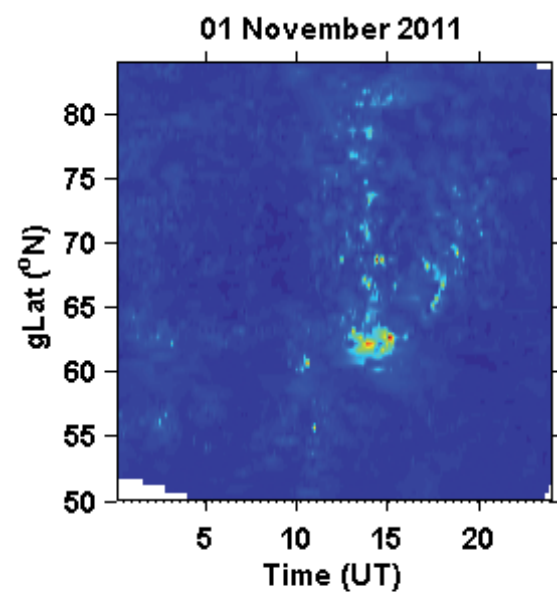

\section{Phi60 (rad)}
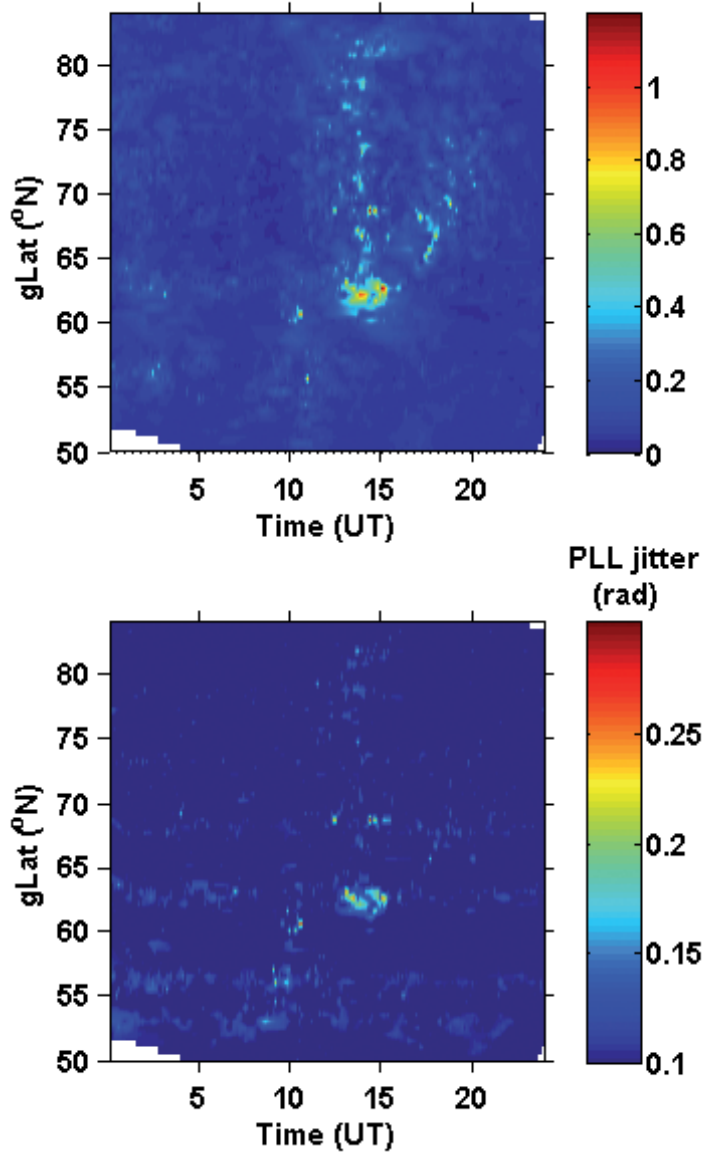

Figure 7. Phase scintillation index $\sigma_{\Phi}$ (a) and receiver PLL jitter maps (b) as functions of UT and IPP geographic latitude for the CHAIN GPS L1 signals. from the OMNI dataset projected to subsolar bow shock (http:/ / omniweb.gsfc.nasa.gov/). The arrival of an ICME on November 1 (shown by a vertical dotted line) resulted in an $A E$ index of about $1000 \mathrm{nT}$ and the $D s t$ index dipped to $-61 \mathrm{nT}$, intensifying a geomagnetic storm that started earlier after the arrival of another ICME and a moderate HSS stream on October 30. The hourly percent occurrence of phase scintillation index $\sigma_{\Phi}>0.1 \mathrm{rad}$ was enhanced between 10:00 and 22:00 UT, as shown for the Cambridge Bay GPS receiver.

Figure 7 shows the maps of $\sigma_{\Phi}$ values (top panel) and PLL jitter (bottom panel) as a function of UT and IPP geographic latitude for the CHAIN GPS L1 signals, as described in the previous section. These maps were constructed using the data from all of the stations of Figure 1, except station Qikiqtarjuaq. It is also worth noting that for the construction of the maps in Figure 7, not only the PLL jitter have been verticalised, but also the $\sigma_{\Phi}$ values. The latter were verticalised using the mapping function described in Spogli et al. [2009]. It can be observed from Figure 7 that the regions of enhanced PLL jitter generally coincide with enhancements in $\sigma_{\Phi}$. The phase scintillation events at high latitudes occurred primarily in the cusp and dayside polar cap between $\sim$ 10:00 and 20:00 UT, which also coincided with the occurrence of PLL jitter enhancements. This indicates increased likelihood for the occurrence of cycle slips and loss of lock, which degrade the positioning accuracy.

In Figure 8 the percentage occurrence of scintillation and PLL jitter are mapped as functions of magnetic latitude and MLT, to show the main regions of enhanced scintillation relative to the auroral oval. Figure 8 shows the occurrence maps for $S_{4}$ and the $\sigma_{\Phi}$ indices, as well as for PLL jitter, on a coarser grid 
(compared to Figure 7 ) of $1^{\circ} \times 0.25 \mathrm{~h}$ of MLT. The position of the statistical auroral oval for very disturbed conditions (IQ = 6) [Feldstein 1967, Holzworth and Meng 1975] is superposed. The phase, and to a lesser degree the amplitude, scintillation occurrence exceeds given threshold values maximized in the cusp and the polar cap, although significant scintillation also occurred at auroral and even at subauroral latitudes. The percentage occurrence of PLL jitter exceeding $0.15 \mathrm{rad}$ was significantly lower, although this generally mapped to the same regions as the phase scintillation; i.e., the cusp, the polar cap, and the auroral oval.

Figure 9 shows the 60-s values of $\sigma_{\Phi}$ and PLL jitter obtained for the CHAIN GPS receiver in Iqaluit on November 1, 2011. The scatter plot (Figure 9c) shows that $\sigma_{\Phi}$ is moderately correlated with the PLL jitter (Pearson's correlation coefficient, 0.68).

\section{Discussion}

The climatology of GPS phase scintillation at high latitudes [Prikryl et al. 2011a] has been extended by two more years to a period from 2008 to 2011. This larger dataset has confirmed that scintillation predominantly occurs in the ionospheric footprint of the cusp. The primary driver of the cusp dynamics is the solar wind coupling to the dayside magnetopause, which peaks with arrivals of ICMEs and HSS / CIRs. Based on the statistics of these solar wind events, using a superposed epoch analysis technique, a probabilistic forecasting method that focuses on the phase scintillation in the cusp has been proposed [Prikryl et al. 2012]. Solar activity has been gradually rising since 2010 , and there was a significant increase in the number of moderate ICMEs in addition to HSS / CIRs. The number of ICMEs that resulted in Dst $<-10 \mathrm{nT}$ doubled in 2011 alone, when compared with the period of extended solar minimum (2008-2010). The superposed-epoch analysis based on improved statistics of events shows consistently higher probability of scintillation occurrence in the cusp on the days of arrival of ICMEs and HSS / CIRs, in contrast to the low probability 1-2 days earlier. This pattern of significant increase in scintillation occurrence makes it possible to specify phase scintillation forecasts for anticipated arrival days of ICMEs and HSS / CIRs [Prikryl et al. 2012].

While the scintillation activity in the polar cap was generally quite low from 2008 to 2010, during moderate to strong events, irregularities in a tongue of ionization or in polar patches that were drawn into the polar cap caused significantly enhanced scintillation occurrence in polar regions [Prikryl et al. 2011b, Prikryl et al. 2013a, this issue]. This depended on the phase of the season in either the southern or northern hemisphere. In partic-
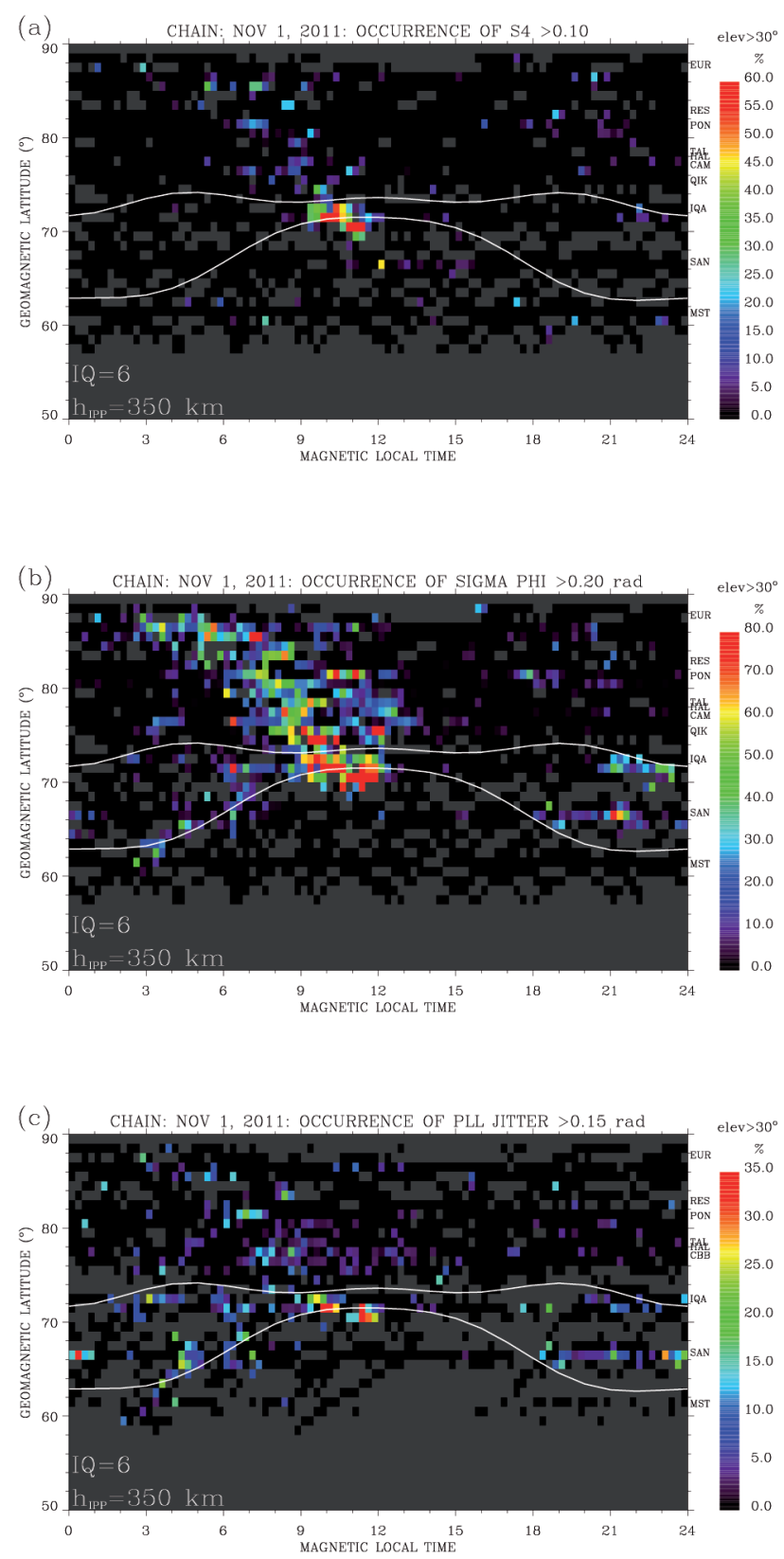

Figure 8. The occurrence of $S_{4}$ index (a), phase scintillation (b) and receiver PLL jitter (c) as functions of MLT and CGM latitude for November 1, 2011. The statistical auroral oval for very disturbed conditions is superposed.

ular, the event discussed in Section 7 was associated with strong ionospheric convection across the polar cap and enhanced TEC in a tongue of ionization [Prikryl et al. 2013b]. Fast drifting ionospheric irregularities within a structured tongue of ionization resulted in high occurrence of strong phase scintillation (Figure $8 \mathrm{~b}$ ).

Under scintillation, maps of tracking jitter [Sreeja et al 2011] can be used to assess the tracking conditions, the likelihood for the occurrence of cycle slips, and the loss of lock, which are known to degrade the GNSS positioning accuracy. The PLL jitter is correlated with $\sigma_{\Phi}$ and the enhancements of PLL jitter appear to coincide, in general, with the occurrence of phase scintillation. 


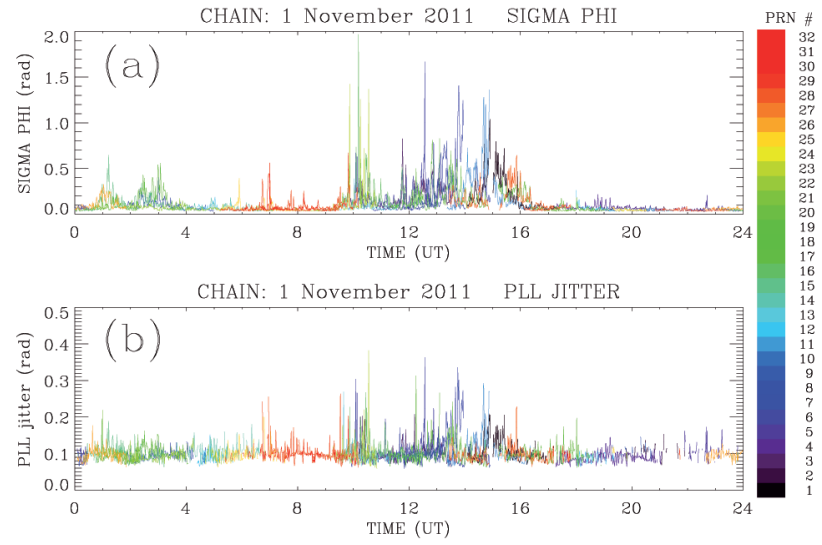

PLL JITTER vs SIGMA PHI

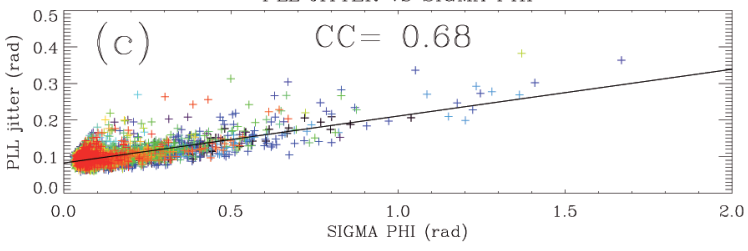

Figure 9. Phase scintillation index $\sigma_{\Phi}(\mathrm{a})$, receiver PLL jitter (b), and correlation between $\sigma_{\Phi}$ and the PLL jitter (c).

Hence, applications relying on robust and accurate phase signal tracking should benefit from the probabilistic forecasting of phase scintillation.

\section{Conclusions}

Phase scintillation occurrence maps from 2008 to 2011 for geomagnetically quiet and disturbed days, and more specifically for the prime days of HSS/CIRs or ICMEs, all show predominance of scintillation occurrence in the cusp. Cumulative probability distribution functions for the phase scintillation occurrence that are a by-product of superposed epoch analysis of growing databases of CIRs and ICMEs can be used in probabilistic forecasting of scintillation activity relative to the arrival times of these major solar wind disturbances. Phase scintillation occurrence can impact on the GNSS receiver tracking performance, which can be assessed by the tracking jitter maps. The PLL jitter is correlated with the phase scintillation index and the regions of enhanced PLL jitter approximately coincide with phase scintillation occurrence. Hence the probabilistic forecasting of scintillation activity should be beneficial in assessing prevailing GNSS tracking conditions at high latitudes, and particularly in the cusp.

Acknowledgements. Infrastructure funding for CHAIN was provided by the Canada Foundation for Innovation and the New Brunswick Innovation Foundation. CHAIN operation is conducted in collaboration with the Canadian Space Agency. Research activities at Nottingham Geospatial Institute, University of Nottingham, are funded by the UK Engineering and Physical Sciences Research Council project Polaris (http: / / www.bath.ac.uk/elec-eng/polaris/) and the FP7 funded project TRANSMIT (http:/ / www.nottingham. ac.uk/transmit/index.aspx). The solar wind data were obtained from Goddard Space Flight Center Space Physics Data Facility OMNIWeb (http:// omniweb.gsfc.nasa.gov/).

\section{References}

Aarons, J. (1982). Global morphology of ionospheric scintillations, Proc. IEE, 70, 360-378.

Aquino, M., M. Andreotti, A. Dodson and H. Strangeways (2007). On the use of ionospheric scintillation indices as input to receiver tracking models, Adv. Space Res., 40, 426-435.

Basu, S., E.J. Weber, T.W. Bullett, M.J. Keskinen, E. MacKenzie, P. Doherty, R. Sheehan, H. Kuenzler, P. Ning and J. Bongiolatti (1998). Characteristics of plasma structuring in the cusp/cleft region at Svalbard, Radio Sci., 33, 1885-1899; doi:10.1029/98RS01597.

Basu, S., K.M. Groves, Su. Basu and P.J. Sultan (2002). Specification and forecasting of scintillations in communication/navigation links: current status and future plans, J. Atmos. Sol.-Terr. Phys., 64, 1745-1754.

Borovsky, J.E., and M.H. Denton (2006). Differences between CME-driven storms and CIR-driven storms, J. Geophys. Res., 111, A07S08; doi:10.1029/2005JA0 11447.

Conker, R.S., M.B. El Arini, C.J. Hegarty and T. Hsiao (2003). Modeling the effects of ionospheric scintillation on GPS/SBAS availability, Radio Sci., 38, 1001; doi:10.1029/2000RS002604.

Feldstein, Y.I., and G.V. Starkov (1967). Dynamics of auroral belt and polar geomagnetic disturbances, Planet. Space Sci., 15, 209-230.

Holzworth, R.H., and C.-I. Meng (1975). Mathematical representation of the auroral oval, Geophys. Res. Lett., 2, 377-380.

Horvath, I., and S. Crozier (2007). Software developed for obtaining GPS derived total electron content values, Radio Sci., 42, RS2002; doi:10.1029/2006RS00 3452.

Jayachandran, P.T., et al. (2009). Canadian High Arctic Ionospheric Network (CHAIN), Radio Sci., 44 (1), RS0A03; doi:10.1029/2008RS004046.

McPherron, R.L., and G. Siscoe (2004). Probabilistic forecasting of geomagnetic indices using solar wind air mass analysis, Space Weather, 2, S01001; doi:10. 1029/2003SW000003.

McPherron, R.L., and J. Weygand (2006). The solar wind and geomagnetic activity as a function of time relative to corotating interaction regions, In: B. Tsurutani, R.L. McPherron, W. Gonzalez, G. Lu, J.H.A. Sobral and N. Gopalswamy (eds.), Recurrent Magnetic Storms: Corotating Solar Wind Streams, AGU Monograph, 167, 125-137.

Osherovich, V.A., J. Fainbergand and R.G. Stone (1999). Solar wind quasi-invariant as a new index of solar activity, Geophys. Res. Lett., 26, 2597-2600.

Prikryl, P., P.T. Jayachandran, S.C. Mushini, D. Pokhotelov, J.W. MacDougall, E. Donovan, E. Spanswick 
and J.-P. St.-Maurice (2010). GPS TEC, scintillation and cycle slips observed at high latitudes during solar minimum, Annales Geophysicae, 28, 1307-1316.

Prikryl, P., P.T. Jayachandran, S.C. Mushini and R. Chadwick (2011a). Climatology of GPS phase scintillation and HF radar backscatter for the high-latitude ionosphere under solar minimum conditions, Annales Geophysicae, 29, 377-392; doi:10.5194/angeo-29-377.

Prikryl, P., et al. (2011b). Interhemispheric comparison of GPS phase scintillation at high latitudes during the magnetic-cloud-induced geomagnetic storm of 5-7 April 2010, Annales Geophysicae, 29, 2287-2304; doi:10.5194/angeo-29-2287.

Prikryl, P., P.T. Jayachandran, S.C. Mushini and I.G. Richardson (2012). Toward the probabilistic forecasting of high-latitude GPS phase scintillation, Space Weather, 10, S08005; doi:10.1029/2012SW000800.

Prikryl, P., Y. Zhang, Y. Ebihara, R. Ghoddousi-Fard, P.T. Jayachandran, J. Kinrade, C.N. Mitchell, A.T. Weatherwax, G. Bust, P. J. Cilliers, L. Spogli, L. Alfonsi, V. Romano, B. Ning, G. Li, M.J. Jarvis, D.W. Danskin, E. Spanswick, E. Donovan and M. Terkildsen (2013a). An interhemispheric comparison of GPS phase scintillation with auroral emission observed at the South Pole and from the DMSP satellite, Annals of Geophysics, 56 (2), R0216; doi:10.4401/ag-6227.

Prikryl, P., R. Ghoddousi-Fard, B.S.R. Kunduri, E.G. Thomas, A.J. Coster, P.T. Jayachandran, E. Spanswick and D.W. Danskin (2013b). GPS phase scintillation and proxy indices observed at high latitudes during a moderate geomagnetic storm, Annales Geophysicae, 31, 805-816; doi:10.5194/angeo-31805-2013.

Richardson, I.G., D.F. Webb, J. Zhang, D.B. Berdichevsky, D.A. Biesecker, J.C. Kasper, R. Kataoka, J.T. Steinberg, B.J. Thompson, C. Wu and A.N. Zhukov (2006). Major geomagnetic storms (Dst $\leq-100 \mathrm{nT}$ ) generated by co-rotating interaction regions, J. Geophys. Res., 111, A07S09; doi:10.1029/2005JA011476.

Richardson, I.G., and H.V. Cane (2010). Near-Earth interplanetary coronal mass ejections during solar cycle 23 (1996-2009): catalog and summary of properties, Solar Phys., 264, 189-237; doi:10.1007/s11207010-9568-6.

Skone, S., and M. de Jong (2000). The impact of geomagnetic substorms on GPS receiver performance, Earth Planets Space, 52, 1067-1071.

Spogli, L., Lu. Alfonsi, G. De Franceschi, V. Romano, M.H.O. Aquino and A. Dodson (2009). Climatology of GPS ionospheric scintillations over high and midlatitude European regions, Annales Geophysicae, 27, 3429-3437.

Sreeja, V., M. Aquino and Z.G. Elmas (2011). Impact of ionospheric scintillation on GNSS receiver tracking performance over Latin America: introducing the concept of tracking jitter variance maps, Space Weather, 9, S10002; doi:10.1029/2011SW000707.

Van Dierendonck, A.J., and B. Arbesser-Rastburg (2004). Measuring ionospheric scintillation in the equatorial region over Africa, including measurements from SBAS geostationary satellite signals, Proc. ION GNSS $17^{\text {th }}$ technical meeting of the satellite division, Long Beach, CA, USA, 316.
${ }^{\star}$ Corresponding author: Paul Prikryl,
Communications Research Centre, Ottawa, Ontario, Canada;
email: paul.prikryl@crc.gc.ca.

(C) 2013 by the Istituto Nazionale di Geofisica e Vulcanologia. All rights reserved. 